\title{
MACROBRACHIUM POTIUNA (MÜLLER): ASPECTOS DO CICLO REPRODUTIVO E SUA RELAÇÃO COM PARÂMETROS AMBIENTAIS (CRUSTACEA, DECAPODA, PALAEMONIDAE).
}

\author{
Yara Maria Rauh Müller ${ }^{1}$ \\ Simone Carpes ${ }^{1,2}$
}

\begin{abstract}
MACROBRACHIUM POTIUNA (MÜLLER): NOTES ON REPRODUCTIVE CYCLE AND IT'S RELATIONS WITH ENVIRONMENTAL FACTORS. Researches under field conditions were done on the breeding cycle of Macrobrachium potiuna (Müller, 1880), a freshwater shrimp from southern Brazil. It was possible to determine the fecundity and establish its relations with the spawn's weight, the total length and the total weight of ovigerous females.

KEY WORDS. Macrobrachium, Palaemonidae, Decapoda, Crustacea, reproductive notes
\end{abstract}

Macrobrachium potiuna (Müller, 1880) é um crustáceo decápodo Palaemonidae exclusivamente endêmico da fauna brasileira (BUENO, 1981). Apresenta uma área de ocorrência subtropical, que vai desde o Espírito Santo até o Rio Grande do Sul(KRETZSCHMAR, 1984; COELHO \& RAMOS PORTO, 1985; BOND-BUCKUP \& BUCKUP, 1989). Os indivíduos são de pequeno porte (KRETZSCHMAR, 1984; VALENTI et al., 1989). GOMES CORRÊA (1977) analisou fêmeas ovígeras com 31 a $50 \mathrm{~mm}$ de comprimento total, e BOND-BUCKUP \& BUCKUP (1989) observaram tais formas ovígeras com 15 a $45 \mathrm{~mm}$ de comprimento. Convém ressaltar que MÜLLER $(1880,1892)$ cita que o amadurecimento sexual nesta espécie inicia-se aos $25 \mathrm{~mm}$ de comprimento total.

De acordo com GOMES CORRÊA (1977) e KRETZSCHMAR (1984), os ovos de $M$. potiuna são grandes e a fecundidade é baixa, podendo ser encontrados até 57 ovos por fêmea ovígera (BOND \& BUCKUP, 1982). As formas ovígeras analisadas por BUENO (1981) apresentaram-se com 24 a 66 ovos em sua câmara incubadora. Segundo BOND \& BUCKUP (1982), à semelhança do que foi observado em outra espécies de crustáceos, em $M$. potiuna o número de ovos constitui uma função linear do comprimento da fêmea.

O ciclo reprodutivo anual de várias espécies do gênero Macrobrachium

\footnotetext{
1) Departamento de Biologia, Universidade Federal de Santa Catarina, Caixa Postal 476, 88049-900 Florianópolis, Santa Catarina, Brasil.

2) Bolsista do CNPq.
} 
tem sido estabelecido por eventuais observações da ocorrência de fêmeas ovígeras. Pouquíssimos autores relacionam a época de oviposição e incubação dos ovos com algum parâmetro ambiental. E tal relação é tão acentuada que entre os camarões do gênero Macrobrachium o ciclo reprodutivo anual pode variar dentro de uma mesma espécie, se forem consideradas regiões geográficas distintas (CARVALHO, 1978). Assim, a mesma autora comenta que estudos que procuram analisar as relações do ciclo sexual com fatores abióticos resultam em informações de importância vital na biologia de qualquer espécie.

BOND \& BUCKUP (1982) consideram que o período reprodutivo de M. potiuna está compreendido desde o mês de agosto até o mês de janeiro. Embora a temperatura venha sendo considerada como um dos fatores mais importantes na regulação do ciclo reprodutivo dos decápodos (CARVALHO, 1978), BOND \& BUCKUP (1982) comentam que o ciclo reprodutivo de $M$. potiuna parece ser estimulado de forma muito mais marcante pelas variações estacionais da luz que pelas flutuações térmicas da água.

Informações precisas sobre o ciclo reprodutivo de M. potiuna, bem como sobre as condições ambientais que nele influem, são dados importantes para uma boa caracterização de sua biologia. Da mesma forma, é importante conhecer também a fecundidade e suas relações com outras variáves inerentes à própria espécie.

\section{MATERIAL E MÉTODOS}

Exemplares de Macrobrachium potiuna foram coletados quinzenalmente numa região entre quedas d'água, conhecida como "Poção" ( $27^{\circ} 37^{\prime} 30^{\prime \prime}$ Lat.S), situada no Córrego Grande, Florianópolis, Ilha de Santa Catarina, no período correspondido de 14 de outubro de 1989 a 15 de abril de 1991.

Os animais foram capturados com o auxílio de um puçá de malha fina. Além da coleta destes, foram registrados dados abióticos do ambiente, tais como as temperaturas do ar e da água, a salinidade e o $\mathrm{pH}$ do ambiente aquático.

Em laboratório, as fêmeas ovígeras foram fixadas em Bouin Alcóolico por 24 horas e, posteriormente, conservadas em álcool $70 \%$. O comprimento total foi obtido medindo-se os exemplares desde a extremidade distal do rostro até a extremidade distal do télson. As fêmeas foram pesadas com os seus ovos ainda na câmara incubadora e depois sem eles, possibilitando o cálculo do peso das ovas. Foi realizada a contagem dos ovos por desova. Conseqüentemente, pôde-se obter o peso aproximado dos ovos individualmente.

Com base nos dados obtidos, foram ajustadas relações entre: fecundidade/peso da ova, fecundidade/comprimento total e fecundidade/peso total. Foi utilizado o Método dos Mínimos Quadrados, com a equação linear $\mathrm{Y}=\mathrm{A}+\mathrm{BX}$. A existência de correlação entre as variáveis foi expressa através do Coeficiente de Correlação Linear de Pearson. 


\section{RESULTADOS}

Durante o período de estudo foram realizadas 40 coletas, onde 515 indivíduos foram capturados. A razão sexual foi de 1,19 sendo que, do total de fêmeas $16,60 \%$ eram formas ovígeras.

As fêmeas ovígeras apareceram pela primeira vez em outubro de 1989 e estiveram presentes até fevereiro de 1990; posteriormente, voltaram a aparecer em setembro de 1990, estando presentes até janeiro de 1991 (Fig. 1). Deste modo, conclui-se que o período de reprodução e desova ocorreu marcadamente nas estações de primavera e verão.

As temperaturas do ar e da água demonstraram uma forte sazonalidade (Fig. 1.A). Ao contrário da salinidade da água (Fig. 1.B) e do pH da mesma (Fig. 1.C), que não demonstraram nenhum padrão oscilatório definido, a temperatura mostrou-se como parâmetro ambiental aparentemente relevante na regulação do ciclo reprodutivo de $M$. potiuna, já que tanto na primeira como na segunda estação reprodutiva o início das mesmas se deu somente quando a temperatura da água estava acima de $18^{\circ} \mathrm{C}$ e a do ar acima de $20^{\circ} \mathrm{C}$.

As fêmeas ovígeras estudadas mediram de 25,40 a $38,35 \mathrm{~mm}$, e pesaram de 0,31 a $1,13 \mathrm{~g}$. Apresentaram uma variação de 1 a 53 ovos, com uma média de aproximadamente 24,4 ovos/fêmea ovígera - quase $50 \%$ destas fêmeas mostraram-se com 20 a 30 ovos em suas câmaras incubadoras. É importante ressaltar que as fêmeas com menos de 15 ovos apresentaram córions vazios junto dos mesmos, sugerindo a eclosão de muitos deles antes das respectivas fêmeas serem capturadas e manuseadas em laboratório. Considerando somente as fêmeas com 15 ou mais ovos, foi obtido um peso médio aproximado de $0,004 \mathrm{~g}$ para cada ovo do camarão estudado.

As relações entre fecundidade/peso da ova (Fig. 2), fecundidade/comprimento total (Fig. 3) e fecundidade/peso total (Fig. 4) são, respectivamente, expressas pelas equações:

$$
\begin{aligned}
& F=13,6541+135,4912 W_{o}, \text { com } r=0,5986 \\
& F=-16,5625+1,4131 \mathrm{~L}, \text { com } r=0,4797 \\
& F=15,1706+21,4117 W, \text { com } r=0,4461
\end{aligned}
$$

Onde: $\mathrm{F}=$ fecundidade

$\mathrm{W}_{\mathrm{O}}=$ peso da ova (em gramas)

$\mathrm{L}=$ comprimento total (em milímetros)

$\mathrm{W}=$ peso total (em gramas)

$\mathrm{r}=$ coeficiente de correlação linear de Pearson

\section{DISCUSSÃO E CONCLUSÕES}

Macrobrachium potiuna apresenta um ciclo reprodutivo anual, intercalado por um período de repouso - fato este observado em quase todas as outras espécies do mesmo gênero (CARVALHO, 1978). O período de reprodução e desova constatado para a região de Poção se assemelha aquele 


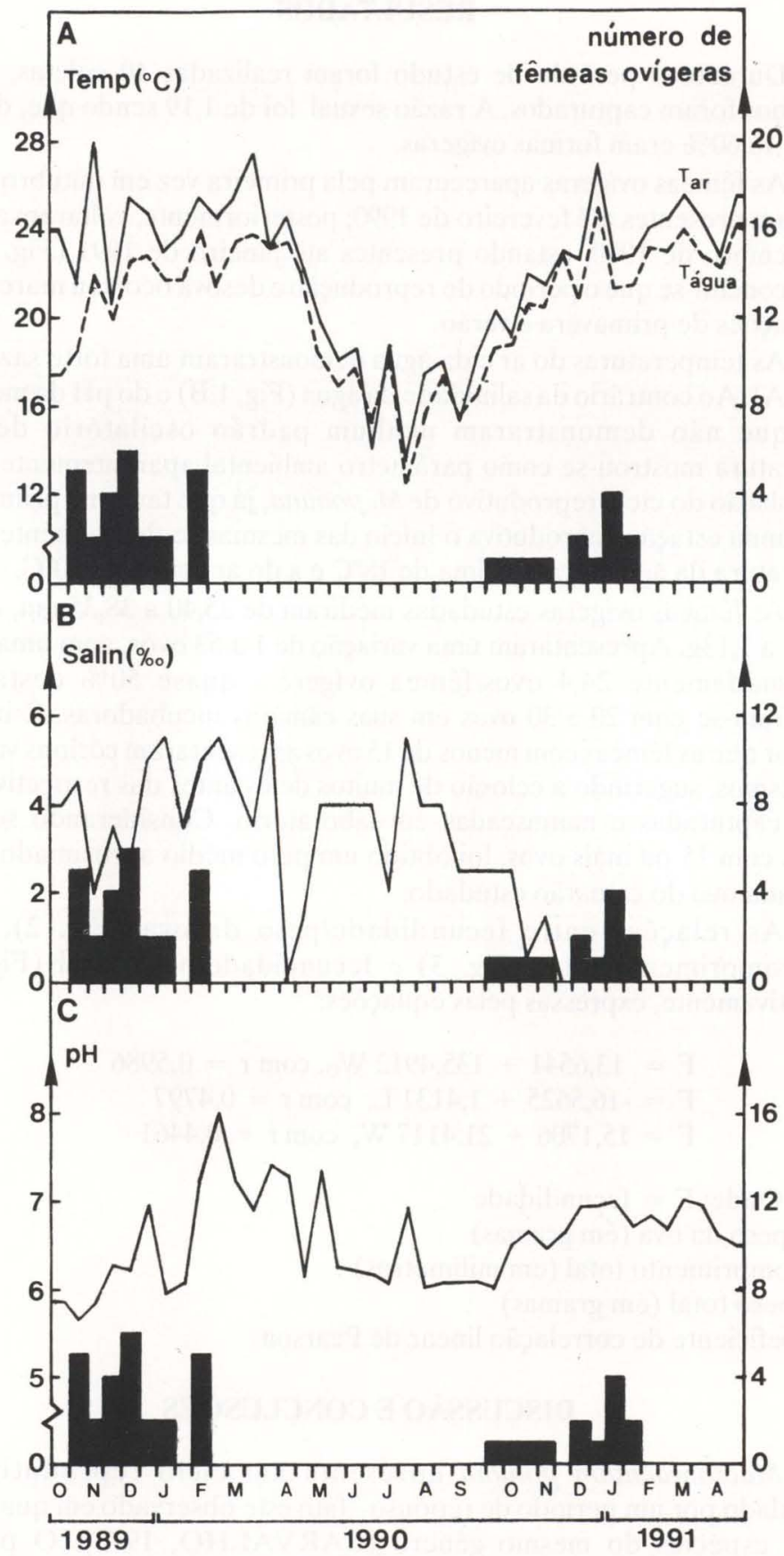

Fig. 1. Registro dos dados abióticos e da presença de fêmeas ovígeras durante o período de estudo. 
considerado por BOND \& BUCKUP (1982) para a espécie, embora seja numa latitude menor do que a do ponto de coleta por eles adotado.

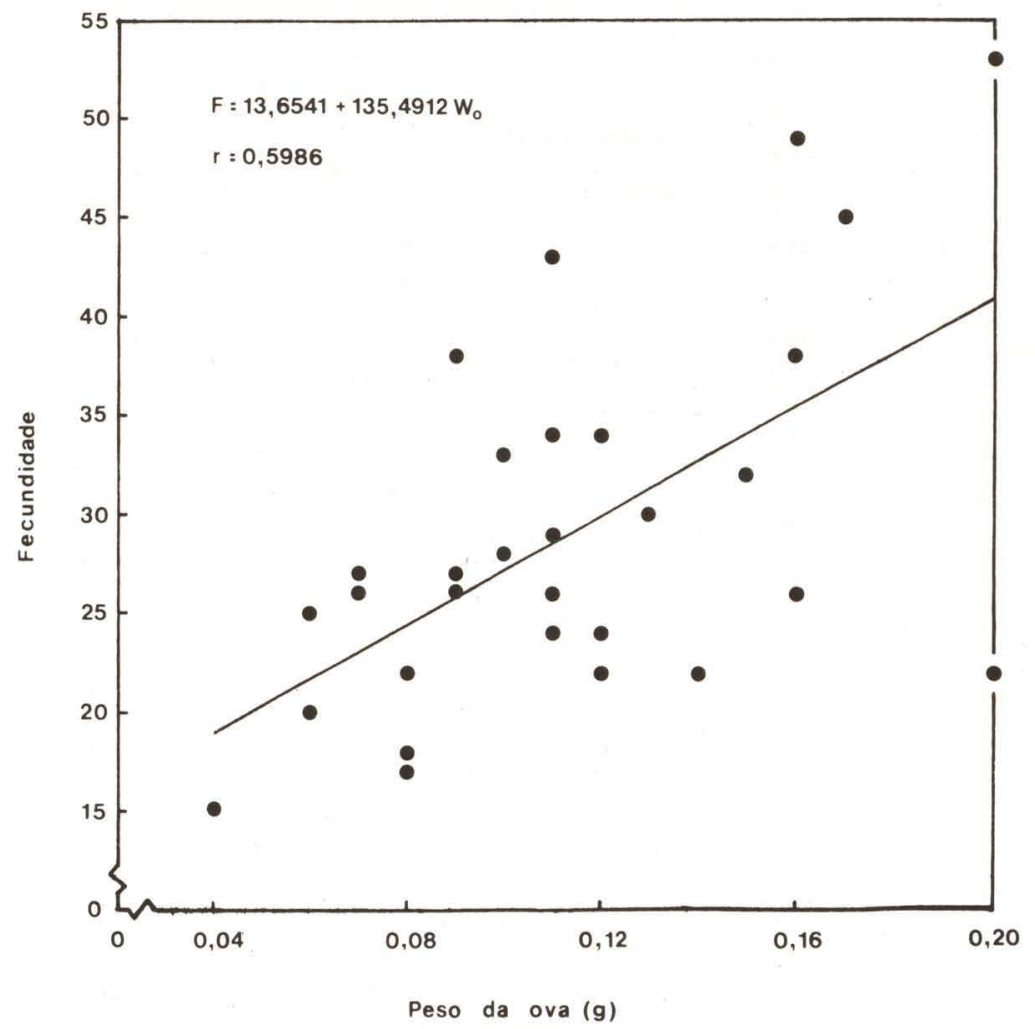

Fig. 2. Relação entre a fecundidade (F) e o peso da ova (Wo).

Pode-se notar que durante o período de estudo houve um deslocamento da segunda estação reprodutiva em relação à primeira, ou seja, o período de reprodução e desova de $M$. potiuna foi antecipado. E, além disso, como o início das duas estações reprodutivas só se deu a partir de determinada temperatura, fica fortalecida a relevância do papel deste fator abiótico na regulação do ciclo reprodutivo da espécie. Como $M$. potiuna é considerado de água doce (IHERING, 1897; HOLTHUIS, 1952; GOMES CORRÊA, 1977; BOND \& BUCKUP, 1982; MOREIRA et al., 1983; KKETZSCHMAR, 1984), isto talvez possa explicar o fato da salinidade aparentemente não ser relevante na regulação do seu ciclo reprodutivo.

Ao contrário de outras espécies de Palaemonidae, os indivíduos de $M$. potiuna analisados se apresentaram com ovos de tamanho considerável e em pouca quantidade, concordando com a bibliografia consultada. Comparando com a mesma, tem-se que os dados de fecundidade mostraram-se mais 
semelhantes aos resultados obtidos por BOND \& BUCKUP (1982), embora estes não tenham realizado nenhum comentário sobre a presença de córions vazios nas câmaras incubadoras. Pelo fato dos ovos serem grandes, apresentaram também um peso considerável.

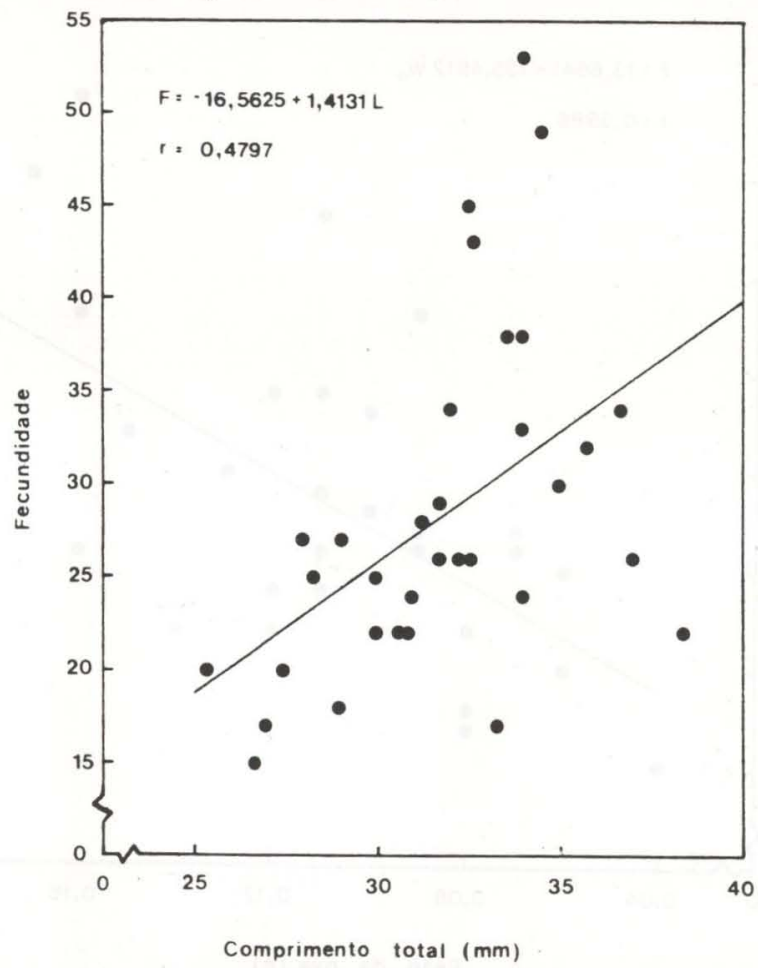

Fig. 3. Relação entre a fecundidade (F) e o comprimento total (L).

Como citado pela bibliografia, pode ser aqui constatado que em $M$. potiuna o número de ovos constitui uma função linear do comprimento da fêmea. A fecundidade também constitui uma função linear do peso da ova e do peso total da fêmea. Resultados semelhantes foram obtidos por VALENTI $e t$ al. (1989) e por MÜLLER el al. (no prelo) para a espécie Macrobrachium acanthurus (Wiegmann, 1836). BOND \& BUCKUP (1982) analisaram apenas relações entre fecundidade e comprimento da fêmea. No presente trabalho, $M$. potiuna demonstrou apresentar graus de correlação linear razoáveis entre a sua fecundidade e as outras variáveis analisadas. Porém, ao se comparar com os coeficientes de correlação linear de outras espécies para as mesmas variáveis (PAIVA \& DA COSTA, 1962; LOBÃO et al., 1985; LOBÃO et al., 1986; VALENTI et al., 1989; MÜLLER et al., no prelo), tem-se que os valores aqui obtidos são, de um modo geral, menores do que os mesmos. As fêmeas com menos de 15 ovos em suas câmaras incubadoras foram excluídas dos procedimentos estatísticos justamente para que não houvesse distorções nas 


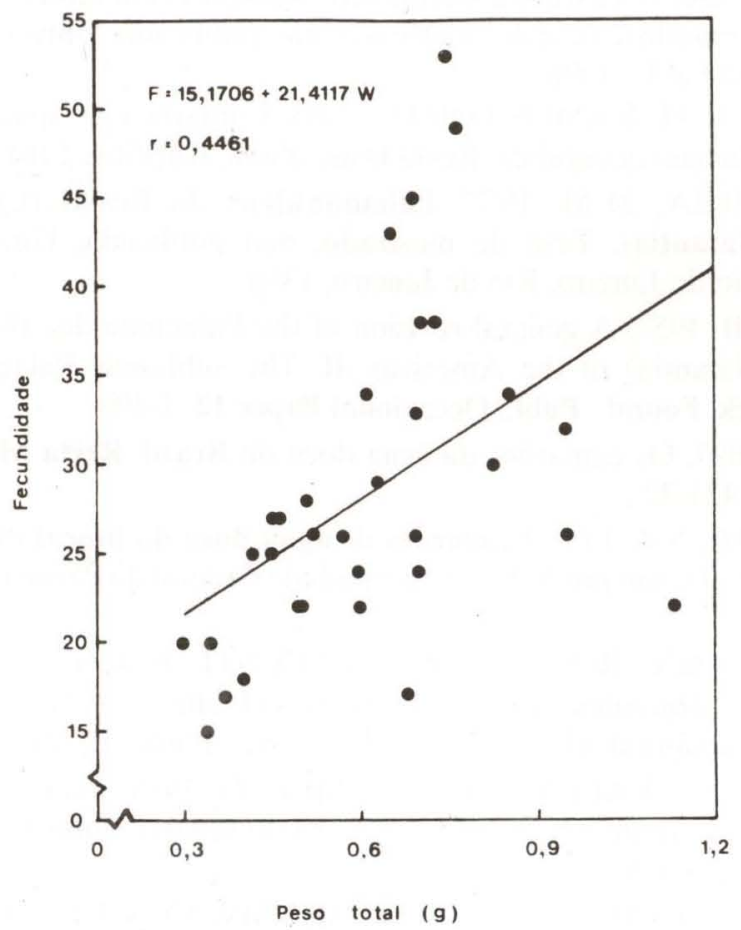

Fig. 4. Relação entre a fecundidade (F) e o peso total (W).

relações estudadas. Assim, o fato dos graus de correlação linear não terem se mosirado elevados talvez possa ser explicado pela baixa fecundidade apresentada por M. potiuna, já que qualquer variação no número de ovos, por menor que seja, pode acarretar em resultados significativos nas análises estatísticas relacionadas à fecundidade da espécie.

\section{REFERÊNCIAS BIBLIOGRÁFICAS}

BOND-BUCKUP, G. \& L. BUCKUP. 1989. Os Palaemonidae de águas continentais do Brasil meridional (Crustacea, Decapoda). Revta bras. Biol., Rio de Janeiro, 49 (4): 883-96.

BOND, G. \& L. BUCKUP. 1982. O clico reprodutor de Macrobrachium borellii (Nobili, 1896) e Macrobrachium potiuna (Müller, 1880) (Crustacea, Decapoda, Palaemonidae) e suas relações com a temperatura. Revta bras. Biol., Rio de Janeiro, 42(3): 473-83.

BUENO, S.L.S. 1981. Desenvolvimento larval de Macrobrachium potiuna (Müller, 1880) e Macrobrachium iheringi (Ortmann, 1897) (Crustacea, Decapoda, Palaemonidae). Tese de mestrado, não publicada, Universidade de São Paulo, São Paulo, 107p.

CARVALHO, H.A. 1978. Ciclo sexual de Macrobrachium acanthurus 
(Wiegmann, 1836) (Crustacea, Decapoda): Relações com fatores abióticos e ciclo de intermudas. Tese de doutorado, não publicada, Universiḍade de São Paulo, São Paulo, 199p.

COELHO. P.A. \& M. RAMOS PORTO. 1985. Camarões de água doce do

Brasil: Distribuição geográfica. Revta bras. Zool., Curitiba, 2 (6): 405-10.

GOMES CORRÊA, M.M. 1977. Palemonídeos do Brasil (Crustacea:

Decapoda: Natantia). Tese de mestrado, não publicada, Universidade

Federal do Rio de Janeiro, Rio de Janeiro, 135p.

HOLTHUIS, L.B. 1952. A general revision of the Palaemonidae (Crustacea,

Decapoda, Natantia) of the Americas. II. The subfamily Palaemonidae.

Allan Hancock Found. Publ., Occasional Paper 12: 1-396.

IHERING, H. 1897. Os camarões da água doce do Brazil. Revta Mus. Paul., São Paulo, 2: 421-32.

KRETZSCHMAR, S.Z. 1984. Camarōes de água doce do litoral do Paraná.

Tese de mestrado, não publicada, Universidade Federal do Paraná, Curitiba, $147 \mathrm{p}$.

LOBÃO, V.L.; N.E.T. ROJAS \& W.C. VALENTI. 1986. Fecundidade e fertilidade de Macrobrachium amazonicum (Heller, 1862) (Crustacea, Decapoda) em laboratório. Bolm Inst. Pesca, São Paulo, 13 (2): 15-20.

LOBÃO, V.L.; W.C. VALENTI \& J.T.C. MELLO. 1985. Fecundidade em Macrobrachium carcinus (L.) do Rio Ribeira do Iguape. Bolm Inst. Pesca, São Paulo, 12 (3): 1-8.

MOREIRA, G.S.; J.C. MCNAMARA; S.E. SHUMWAY \& P.S. MOREIRA. 1983. Osmoregulation and respiratory metabolism in Brazilian Macrobrachium (Decapoda, Palaemonidae). Comp. Biochem. Physiol. 74 A (1): 57-62.

MÜLLER, F. 1880. Palaemon Potiuna. Ein Beispiel abgekürzter Verwandlung. Zool. Anz., 3: 152-57.

1892. O camarão preto, Palaemon Potiuna. Arch. Mus. Nac., Rio de Janeiro, 8: 179-206.

MÜLLER, Y.M.R.; C.M. BRESSAN \& E.M. NAZARI. (no prelo). Dados de fecundidade de Macrobrachium acanthurus (Decapoda, Palaemonidae) do Rio Capivari, Praia dos Ingleses, Florianópolis/SC. Biotemas, Florianópolis PAIVA, M.P. \& R.S. DA COSTA. 1962. Sobre os ovos de Macrobrachium acanthurus (Wiegmann, 1836). Pearse, 1911. Bolm Sco. Cearense Agron., Fortaleza, 3: 37-40.

VALENTI, W.C.; J.T.C. MELLO \& V.L. LOBÃO. 1989. Fecundidade em Macrobrachium acanthurus (Wiegmann, 1836) do Rio Ribeira de Iguape (Crustacea, Decapoda, Palaemonidae). Revta bras. Zool., Curitiba, 6 (1): 9-15. 